\title{
A Successful Case Study on Ayurvedic Management of Hypothyrodism
}

\author{
Dnyaneshwar Kantaram Jadhav* \\ Department of Kaychikitsa, Shri Dhanwantri Ayurvedic Medical College \& Research Centre, India
}

Submission: September10, 2019; Published: October 1, 2019

*Corresponding author: Dnyaneshwar Kantaram Jadhav, Department of Kaychikitsa, Shri Dhanwantri Ayurvedic Medical College \& Research Centre, India

\begin{abstract}
We all know that Ayurveda is life science, which has solutions for all heath related issues. Hypothyroidism is one of the most common endocrine disorder seen in daily opd. As per charak samhita we can categorize Hypothyroidism in Anukta vhadhies. Vata and kapha are two main doshas involved in this vhadhies. Present case is one of my successful case of hypothyroidism. 32 year's male patient suffering from weakness, dry skin, poor memory, hair loss, constipation, acidity, breathlessness since last 8 years, and from last one month all symptoms increases rapidly. Patient having history of diabetes and thyroid and under allopathic treatment since last 8 years. After 1.5 months Ayurvedic treatment patient got relief in most of symptoms of disease and TSH level reduced from 18.71 to 3.05.
\end{abstract}

Keywords: Subclinical hypothyroidism; Anukta vhadhie; Ayurved

\section{Introduction}

Luxury lifestyle give pleasure to body. At the same time this pleasure disturbs doshas and ultimate disturb mind and body too. This luxury life give rise to many lifestyle disorders. Thyroid, hypertension, diabetes some examples of lifestyle disorder. In society number of cases of thyroid increases day by days. There are 3.8-6\% general population affected with hypothyroidism [1]. Thyroid dysfunction is two types i.e over activity and under activity. In Hypothyroidism thyroid gland does not produced enough thyroid hormone [2]. Hypothyroidism can be described as underactive thyroid. Infertility, weight problems, depression and chronic tiredness the most frequent complications of hypothyroidism [3]. Ayurvedic Samhita has description of swelling of the thyroid gland called as Galganda which symptoms is like hyperthyroidism [4]. There is not a direct description of hypothyroidism found in samhitas. There are many diseases which is not mentioned directly in Ayurveda texts is called as Anukta vicar [5]. Subclinical hypo-thyroid is one of such disease. Though there is lack of direct description of such disease, but its line of treatment described very well. There is description in Ashtanag hrudaya that, if we don't know name of the disease than Vaidya should treat the patient with examination of prakruti, adhistahna, bheda, hetu [6]. According to Ayurveda Hypothyroidism can be consider as Anukta vikara and discussed as vata-kafaj dushti-janya vhadhi. Modern science is very well developed with advanced techniques for disease, diagnosis and treatment, but still no easy solution to treat lifestyle disease. Current available medicines are even not capable to give relief on such diseases. While Ayurveda has solution on direct describe and indirect (Anukta vikar) described diseases too.

\section{A Case Report}

A 32-year-old male patient came to us with chief compliant of [7]

- $\quad$ Drubalya (Weakness).

- $\quad$ Twak rukshata (Dry skin)

- $\quad$ kesh patana (hair loss).

- malavashtmbha (constipation)

- $\quad$ amalapitta (acidity)

- $\quad$ shwasaKashthta (breathlessness)

- $\quad$ smriti alpata (poor memory)

Patient had above complaints in the last 8 years. $\mathrm{k} / \mathrm{c} / \mathrm{o}$ Dm (for 7 years) (insulin. mixsl 30-70) (36 unit at morning \& 40 units at night), (tab.vogli 0.2 at afternoon) No H/o / HTN, Asthma

\section{History of Personal Illness}

The patient is k/c/o Dm with history of hypothyroidism from last 8 years. He was under modern medicine (insulin. mixsl 30-70) (36 unit at morning \& 40 units at night), (tab. vogli 0.2 at after- 
noon). Even consumption of modern medicine, patient have been suffering from Drubalya (Weakness), Twak rukshata (Dry skin), kesh patana (hair loss), malavashtmbha (constipation), amalapitta (acidity), shwasaKashthta (breathlessness), Smriti alpata (poor memory) Even all medicine this symptoms increased gradually, then he decide to take Ayurveda treatment. For Ayurvedic treatment he came to our clinic - Nakshtra Ayurved Panchkarma Clinic \& Research Center, Mumbai.

\section{Ashtavidha Parikshana}

- $\quad$ Nadi (pulse) $=86 / \mathrm{min}$.(kapha-vata)

- $\quad$ Mala $($ stool $)=$ Malavshtmbha

- $\quad$ Mutra (urine) = Normal

- $\quad$ Jeeva (tounge) = saam

Table 1 : Showing material used for study.

\begin{tabular}{|c|c|c|c|c|}
\hline SR NO & DRAVYA & DOSE & DURATION & ANUPANA \\
\hline 1 & Laghumalini vasant vati & $200 \mathrm{mg}$ & \multirow{3}{*}{500 mg capsule bid } & \multirow{3}{*}{ Luke warm water } \\
\hline 2 & Arogyavardhini vati & $200 \mathrm{mg}$ & & \\
\hline 3 & Vanga bhasma & $15 \mathrm{mg}$ & & \\
\hline 4 & Abharak bhasma & $15 \mathrm{mg}$ & \multirow{3}{*}{500 mg capsule bid } & \multirow{3}{*}{ Luke warm water } \\
\hline 5 & Kanchanaargugul & $250 \mathrm{mg}$ & & \\
\hline 6 & Chandraprabha vati & $250 \mathrm{mg}$ & & \\
\hline 7 & Gandhrva haritaki & $500 \mathrm{mg}$ & H. S & Luke warm water \\
\hline
\end{tabular}

\section{Methods}

Centre of study: Nakshtra Ayurved panchkarma clinic \& research center, Mumbai.

Type of study: Simple random single case study.
- $\quad$ Agni $=$ Kshudhamandya

- $\quad$ Shabda $($ speech $)=$ Normal

- $\quad$ Sparsha $($ skin $)=$ Twak rukshata

- $\quad$ Druka (eyes) = upanetra

- $\quad$ Akruti $=$ Madhyama

- $\quad$ Bala $=$ Madhyama

- $\quad$ Raktadaaba (B.P) $=125 / 90 \mathrm{~mm} / \mathrm{Hg}$

\section{Materials and Method}

\section{Materials}

(Table 1)

\section{Observations and Results}

(Table 2,3) Due to our Ayurvedic management there are revealed Regression of symptoms. The patient had started improving symptoms within 7 days. After 1.5 months treatment patient cured subjective as well as objective.

Table 2 : showing daily treatment with prognosis.

\begin{tabular}{|c|c|c|c|c|c|}
\hline DAYS Symptoms & $\mathbf{1}^{\mathrm{ST}}$ (after 7 days) & $\mathbf{2}^{\mathrm{ND}}$ (after 14 days) & $\mathbf{3}^{\mathrm{RD}}$ (after 21 days) & $\mathbf{4}^{\mathrm{TH}}$ (after 28 days) & $\mathbf{5}^{\mathrm{TH}}$ (after 35 days) \\
\hline $\begin{array}{c}\text { Drubalya } \\
\text { (Weakness) }\end{array}$ & ++ & ++ & ++ & 0 \\
\hline $\begin{array}{c}\text { Twak rukshata } \\
\text { (Dryskin) }\end{array}$ & ++ & ++ & + & 0 \\
\hline $\begin{array}{c}\text { kesh patana } \\
\text { (Hair loss) }\end{array}$ & ++ & ++ & 0 & 0 \\
\hline $\begin{array}{c}\text { malavashtmbha } \\
\text { (constipation) }\end{array}$ & ++ & 0 & 0 & 0 \\
\hline $\begin{array}{c}\text { amalapitta } \\
\text { (acidity) }\end{array}$ & + & 0 & 0 & 0 \\
\hline $\begin{array}{c}\text { shwasaKashthta } \\
\text { (breathlesness) }\end{array}$ & + & + & + & 0 \\
\hline $\begin{array}{c}\text { smriti alpata } \\
\text { (poor memory) }\end{array}$ & + & + & 0 \\
\hline
\end{tabular}

Table 3 : Showing changes in thyroid reports

\section{THYRIOD PROFILE}

\begin{tabular}{|c|c|c|c|}
\hline \multicolumn{5}{|c|}{ THYRIOD PROFILE } \\
\hline & BEFORE (9/4/2018) & AFTER (16/6/2018) & NORMAL RANGE \\
\hline TSH & 18.71 & 3.05 & $0.3-5.5$ \\
\hline
\end{tabular}




\section{Discussion}

\section{Hetu of subclinical-Hypothyriodism}

\section{Ahar}

i. Improper and irregular diet.

ii. Chiken, Matsya sevan (3 times in week )

iii. Dadhi (twice in week)

iv. Biscuit (daily with milk)

v. Dosa, idali ,sandwich (daily)

vi. Daily empty stomach water (4-5 glass per day)

\section{Vihar}

i. Prolong seating

ii. Ratri jagran

iii. Excessive sex/ Mastrubation

Manasika nidan: Chinta, vegavrodha causes vata vrudhi

\section{Sanprapti Ghatak}

- Dosh: vata-kapha.

- $\quad$ Dushya: Rasa, Rakta, shukra

\section{Vikalpa Samprapti}

- $\quad$ Vata dosha- sheeta guna [8]

- $\quad$ Kapha dosha- manda, sheeta, guru

\section{Samprapti}

All hetues in this case are mainly vata and kafa prakopak [9]. Due to hetu-sevan aamuttpatti occurs which causes agnimandya and constipation. Due to hetues first aahar-rasa convert into Aam, which make vikrut rasa dhatu. All this further disturb and produce vikrut dhatu-uttpatti. Excessive sex and Mastrubation like cause direct act on shukra dhatu and shown its symptoms. It also does dhatu kshaya via pratilom gati and show as per symptoms [10-16]. (Flow chart)

Diagnosis of hypothyroidism with its symptoms along with blood test measuring T3, T4, TSH levels.(Table 4)

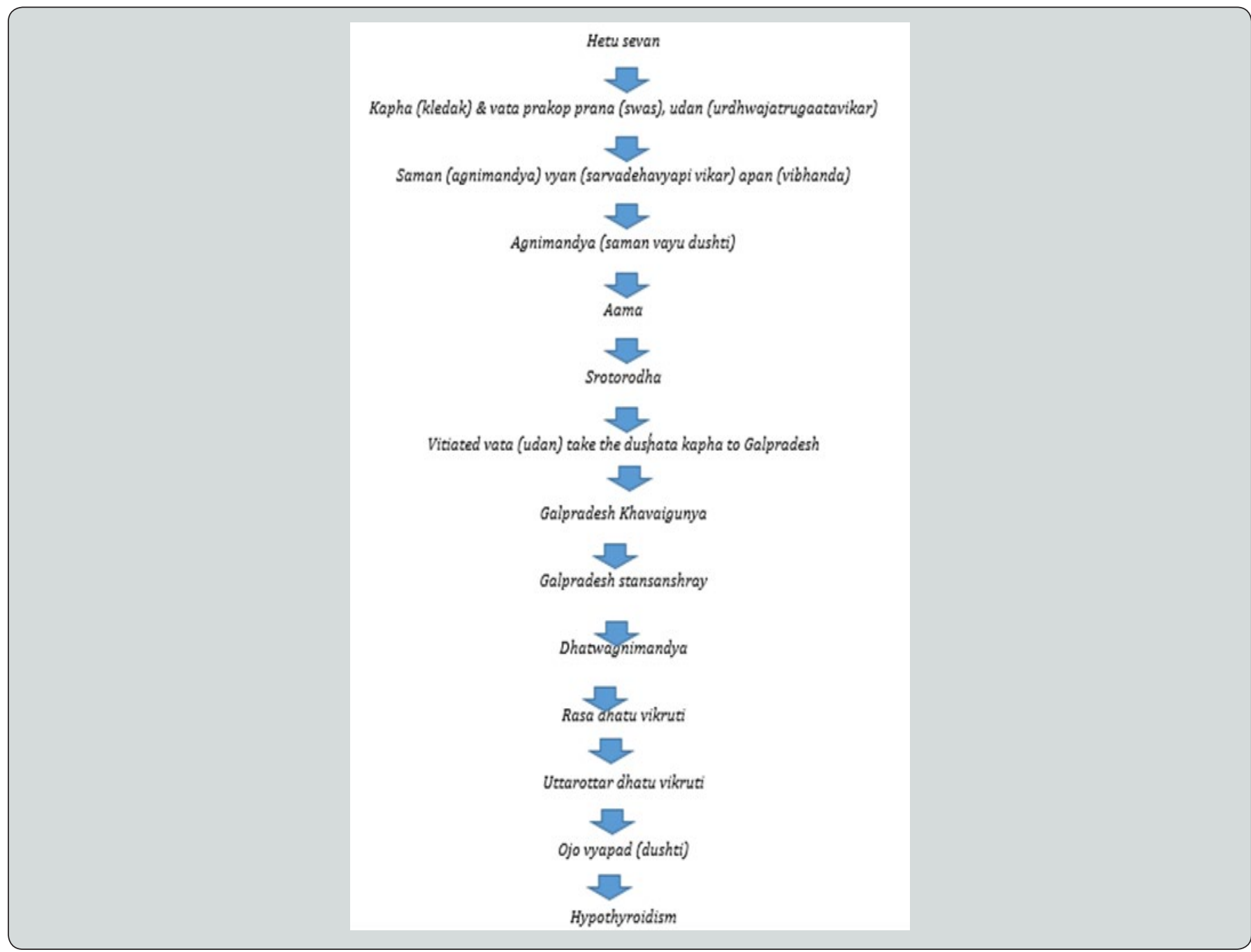


Table 4 : : showing how to Diagnosis of hypothyroidism with T3,T4,TSH levels.

\begin{tabular}{|c|c|c|}
\hline TSH & T4 & INTERPRETATION \\
\hline Normal & Normal & Normal thyroid function \\
\hline Elevated & Low & central hypothyroidism \\
\hline Normal & Low & subclinical hypothyroidism \\
\hline Elevated & Normal \\
\hline
\end{tabular}

Table 5: Showing action of drug \& other procedure done in study.

\begin{tabular}{|c|c|c|}
\hline SR.NO & DRAVYA & ACTION \\
\hline 1 & Laghumalini vasant vati [10] & Rasayana mainly Work on rasa-rakta dhatu, Balya, dipan, panchan. \\
\hline 2 & Arogyavardhini vati [11] & Dhatwagnivardhana, malashodhaka, Pakwashyadushti nashka. \\
\hline 3 & Vanga bhasma [12] & Mehaghan, shleshmaghna, balya, prushti, vrushay, pachana \\
\hline 4 & Abharak bhasma [13] & Vatakaphagha, hrudya, balya, Dhatuposahk, dipan, pachana, vrushay \\
\hline 5 & Kanchanaar guggul [14] & Kaphaghan \\
\hline 6 & Chandraprabha vati [15] & Tridoshaghana, balya, vrushya, rasayana,dhatuposhak, agnimadhyanashak, shamak, hrudya \\
\hline 7 & Gandhrva haritaki $[16]$ & Anulomna, vatashulnashak \\
\hline
\end{tabular}

Action of Drug \& Other Procedure in Management of Subclinical- Hypothyriodism

(Table 5)

\section{Conclusion}

Lifestyle disorders becomes headache due its cases increased day by days. Ayurveda has best solution in such type of Anukta vhyadhi. With doshas vichar, Ayurveda cure such disease \& act as apunarbhava also. This is one example of successful case of subclinical hypothyroid among my all cured thyroid case. I will work out further research on same disease.

\section{References}

1. https://en.m.wikipedia.org/wiki/Lifestyle_disease

2. Harsh mohan (Ed.) Textbook of pathology ( $5^{\text {th }}$ edn). Jaypee publication, New Delhi, India, pp. 827.

3. Sandhya Kamath (Ed.) APT Textbook of medicine ( $7^{\text {th }}$ edn). published by association of physician of India, India, pp. 1051.

4. Brhamananda Tripathi (Ed.) Charak Samhita of maharshi charak, Charakacnahdrika commentatory, Charaka samhita part 1 , ( $5^{\text {th }}$ edn $)$. Chaukambha Sanskrit sansthana, Varanasi, Uttar Pradesh, India, pp. 372.

5. Brhamananda Tripathi (Ed.) Charak Samhita of maharshi charak, Charakacnahdrika commentatory, Charaka samhita part $1,\left(6^{\text {th }}\right.$ edn $)$. Chaukambha Sanskrit sansthana, Varanasi, Uttar Pradesh, India, pp. 378.
6. Ganesh krusha sarth vagbhat ( $1^{\text {st }}$ edn). profeshant publication, pp. 67.

7. Sahu Dustidev, Gupta Mahesh Chand, Indoria Anooopkumar (2015) Hypothyroidism an Ayurvedic Perspective - A Critical Review. International Ayurvedic Medical Journal 3(1): 152.

8. Vd Gopakumar (Ed.) Samsidhi- Gk's book on classical thoughts and clinical targets, hypothyroidism ( $4^{\text {th }}$ edn). mylandoor ayurvedic research center, kerala, India, pp. 108-115.

9. Rangarajan, N Ashwini, Muralidhara (2018) A Ray of Hope to Hypothyroidism: A Case Study: International Journal of Applied Ayurved Research 3(9): 1372.

10. Gune GP Vd (2005) Aushodhigunadharmashtri, part-4, kalpa no-78, India, pp. 440.

11. Gune GP Vd (2005) Aushodhigunadharmashtri, part-2, kalpa no-10, India, pp. 208.

12. Gune GP Vd (2005) Aushodhigunadharmashtri, part-1, kalpa no-13. India, pp. 123.

13. Gune GP Vd (2005) Aushodhigunadharmashtri, part-1, kalpa no-1. India, pp. 19.

14. Brhamananda Tripathi (Ed.) Sharangdhar-samhita of pandit sarangadharacaya (with dipika hindi commentary), madhyama khanda, Chukhambha prakashan, Varanasi, Uttar Pradesh, India, pp. 207.

15. Gune GP Vd (2005) Aushodhigunadharmashtri, part-3, kalpa no-39, India, pp. 311.

16. https://www.bimbima.com/ayurveda/gandharva-haritaki-churnabenefits-ingredients-dosage/466/ 

(C) (i) $\begin{aligned} & \text { This work is licensed under Creative } \\ & \text { Commons Attribution 4.0 Licens }\end{aligned}$ BY DOI: 10.19080/JETR.2019.05.555654

\section{Your next submission with Juniper Publishers} will reach you the below assets

- Quality Editorial service

- Swift Peer Review

- Reprints availability

- E-prints Service

- Manuscript Podcast for convenient understanding

- Global attainment for your research

- Manuscript accessibility in different formats ( Pdf, E-pub, Full Text, Audio)

- Unceasing customer service

Track the below URL for one-step submission https://juniperpublishers.com/online-submission.php 